\title{
Postoperative Endocrine Therapy for Invasive Breast Cancer
}

\author{
Leisha A. Emens and Nancy E. Davidson \\ Breast Cancer and Tumor Immunology Programs, Department of Oncology, The Johns Hopkins \\ University School of Medicine and the Sidney Kimmel Comprehensive Cancer Center at Johns \\ Hopkins, 1650 Orleans Street, Room 409, Bunting Blaustein Cancer Research Building, \\ Baltimore, MD 21231-1000, USA \\ Leisha A. Emens: emensle@jhmi.edu
}

\section{Introduction}

Estrogen plays a key role in mammary carcinogenesis, identifying the pathways that regulate estrogen function as major targets for breast cancer therapy. Expression of the estrogen receptor $\alpha(\mathrm{ER} \alpha)$ or the progesterone receptor $(\mathrm{PR})$ is a widely recognized predictor of response to hormonal therapy [1]. Studies of endocrine manipulation conducted in advanced breast cancer have revealed response rates of $80 \%$ for ER+/PR+ tumors, $40-45 \%$ for ER-/ $\mathrm{PR}+$ tumors, $25-30 \%$ for ER+/PR - tumors, and less than $10 \%$ for ER-/PR - tumors. More recently, data have demonstrated that over-expression of ER or PR in at least $1 \%$ of breast tumor cells indicates potential responsiveness to endocrine therapy [2,3]. In the absence of ER or PR expression, endocrine therapy is not indicated. These observations highlight the importance of an accurate measurement of ER and PR expression in the primary tumor in therapeutic decision-making. Currently, quality-controlled quantitative immunohistochemistry is the method of choice $[4,5]$.

The estrogen pathway can be manipulated by several distinct strategies. Estrogen production itself can be decreased by ovarian ablation/suppression [6] or aromatase inhibitors [7]. Alternatively, ER $\alpha$ activity can be modulated using Tamoxifen or other selective estrogen receptor modulators (SERMs) like Raloxifene [8]. Furthermore, the ER $\alpha$ can be downregulated by Fulvestrant, a selective estrogen receptor destroyer (SERD) [9]. Ovarian function has a major impact on circulating levels of estrogen, and menopausal status drives decision-making when choosing endocrine therapies for women with invasive breast cancer. In premenopausal women, the ovary is the major source of estrogen production. A smaller amount of estrogen is produced by extragonadal aromatase, which catalyzes the conversion of androstenedione and testosterone to estrone and estradiol respectively. In postmenopausal women, peripheral aromatization is the primary source of estrogen. Therefore, ablating ovarian function by surgical removal, targeted medical suppression, or the indirect effects of chemotherapy is most effective for decreasing estrogen levels in women with intact ovarian function. In contrast, the prevention of extragonadal estrogen production by inhibiting aromatase is the most effective means of decreasing estrogen levels in postmenopausal women. Importantly, the most recent Early Breast Cancer Trialists' Collaborative Group (EBCTCG) overview analysis demonstrated that 5 years of adjuvant Tamoxifen reduced the annual rate of death by $31 \%$ among women with ER+ breast cancer, regardless of age [10]. However, recent advances in breast cancer biology have highlighted the heterogeneity of the disease, with gene expression profiles classifying luminal A and luminal B as two distinct

(C) Springer Science+Business Media, LLC 2009

Correspondence to: Leisha A. Emens, emensle@ jhmi . edu. 
biological subtypes of ER+ breast cancer with very different natural histories [11-13]. Further, multiple mechanisms of resistance to endocrine therapy can account for Tamoxifen failure. These include de novo resistance pre-determined by tumor cell biology [14], acquired resistance that develops over time with treatment [15], genetic resistance related to inherited differences in drug metabolism [16], or unresponsive estrogen/progesterone receptor variants [17]. These findings, together with both the emergence of new drugs and the resurgence of old therapies, highlight the complexity of treatment decision-making for women with ER+ disease. Here, we present an overview of the current data guiding the use of endocrine manipulation for early breast cancer treatment, and highlight emerging opportunities for further refinement.

\title{
Adjuvant Endocrine Therapy for Premenopausal Women with Early Breast Cancer
}

\begin{abstract}
About one quarter of newly diagnosed breast cancers occur in women under 50 years of age, a commonly used surrogate for premenopausal status [18]. About $60 \%$ of these young women have hormone-responsive tumors [19], and have typically been offered adjuvant Tamoxifen therapy with or without ovarian suppression [20]. Historically, many of these premenopausal women with disease that is potentially hormone-sensitive have also received adjuvant chemotherapy, particularly if there is a high risk of relapse (large tumors or positive lymph nodes) [4]. The use of chemotherapy in women with lymph node-negative ER+ disease has been more controversial. Much of the benefit of chemotherapy may be due to chemotherapy-induced ovarian suppression rather than to the direct cytotoxic effects of chemotherapy in this patient population. These uncertainties have led to two major research initiatives. First, defining the role of ovarian ablation in the management of premenopausal women with early breast cancer is the goal of several ongoing international clinical trials [21]. Second, the development of techniques to define better the relative responsiveness of breast cancers to endocrine manipulation and/or chemotherapy has recently led to a shift in assessing recurrence risk based on tumor biology in addition to tumor size and lymph node status [22-24].
\end{abstract}

\section{Tamoxifen Alone}

The 1995 EBCTCG overview showed that 5 years of Tamoxifen given to women younger than 50 years old resulted in proportional risk reductions in recurrence and mortality of 45 and 32\% respectively [25]. A number of randomized trials have defined the role of Tamoxifen in this patient population. The Nolvadex Adjuvant Trial Organization (NATO) [26], the National Surgical Adjuvant Breast and Bowel Project (NSABP) B-14 [27, 28], and the Scottish trials [29-31] tested Tamoxifen therapy alone against observation or placebo. The NSABP and Scottish studies re-randomized patients who remained disease- free after 5 years of Tamoxifen to either stop Tamoxifen or continue it for 5 additional years. All of these clinical trials revealed a substantial benefit for 5 years of Tamoxifen therapy (Table 1). Longer therapy was associated with an increased risk of endometrial cancer in one trial [31], but no additional clinical benefit in any trial. Therefore, 5 years of adjuvant Tamoxifen therapy is currently the standard of care for premenopausal women with hormone receptorpositive early breast cancer.

\section{Tamoxifen plus Chemotherapy}

The EBCTCG overview revealed proportional risk reductions of the annual mortality rate for premenopausal women with ER+ breast cancer treated with anthracycline-based chemotherapy plus tamoxifen compared to anthracycline-based chemotherapy alone of 57 and $38 \%$ respectively [10]. Tamoxifen has been compared with chemotherapy by the Italian 
Breast Cancer Adjuvant Study Group (GROCTA) [32] and by the Gynecological Adjuvant Breast Group (GABG) [33]. Although there was no statistically significant difference in outcome in the GROCTA study, those in the GABG study treated with chemotherapy (CMF) had more favorable outcomes (disease free survival (DFS) and overall survival (OS)) than those treated with Tamoxifen alone [33] (Table 1). The Eastern Cooperative Oncology Group ECOG conducted a clinical study testing the efficacy of a doxorubicin-based regimen and 5 or more years of tamoxifen in 533 premenopausal women with lymph node-positive disease; 335 women expressed ER $\alpha$ and 198 women did not. This study demonstrated improved 5 year DFS with the addition of at least 5 years of tamoxifen to chemotherapy for the patients with ER+ disease (78\%) compared to those with ER-negative disease (58\%) $(\mathrm{p}=0.03$ vs $\mathrm{p}=0.63$ ) [34]. The International Breast Cancer Study Group (IBCSG) Trial 13-93 enrolled 1,246 pre- and perimenopausal patients with lymph node-positive disease, randomizing them to receive chemotherapy alone, or chemotherapy followed by 5 years of Tamoxifen [35]. In this study the addition of Tamoxifen to chemotherapy improved DFS compared to chemotherapy alone in patients with ER+ breast cancers, with a hazard ratio of 0.59 ( $\mathrm{p}<0.0001)$. Tamoxifen was ineffective in those breast cancers classified as ER-, with a hazard ratio of $1.02(\mathrm{p}=0.89)$. An unplanned exploratory analysis revealed that patients with tumors completely devoid of ER expression had a seemingly detrimental effect with the addition of Tamoxifen to chemotherapy, with a hazard ratio of $2.10(\mathrm{p}=0.04)$. Interestingly, those patients with ER+ tumors who experienced chemotherapy-induced amenorrhea had a significantly improved outcome compared to those without chemotherapy-induced amenorrhea $(\mathrm{HR}=0.61$ for amenorrhea compared to no amenorrhea, $\mathrm{p}=0.004)$, regardless of whether Tamoxifen therapy was used. This finding highlights the potential importance of directly modulating ovarian estrogen production as primary breast cancer therapy.

\section{Ovarian Ablation/Suppression Alone}

The ovaries are the major source of estrogen production in premenopausal women, with a smaller amount of estrogen derived from the peripheral aromatization of androgens. Thus, oophorectomy or ovarian irradiation has been used to ablate ovarian function as breast cancer therapy for over 100 years. Beatson first reported the use of ovarian ablation in the palliation of young women with breast cancer in 1896 [36]. More recently, luteinizing hormone releasing hormone (LHRH) analogues have been used to manipulate ovarian function. These drugs impinge on the hypothalamic-pituitary-ovarian axis to suppress circulating levels of estrogen to post-menopausal levels. Medical suppression has the advantage of lower morbidity, with the potential for restoration of ovarian function upon drug withdrawal to maintain fertility in young premenopausal women. Generally, ovarian ablation and medical ovarian suppression (OA/OS) are considered to have equivalent activity in reducing ovarian function.

Randomized trials of adjuvant ovarian ablation/suppression began in 1948 [6]. The combined analysis of these trials by the EBCTCG conclusively demonstrated that ovarian ablation alone decreases breast cancer recurrence and increases survival in women less than 50 years old [10]. Ovarian ablation/suppression vs not in ER+ breast cancer resulted in an absolute decrease in the 15-year recurrence and mortality rates of $4.3 \%(\mathrm{p}=0.00001)$ and $3.2 \%(\mathrm{p}=0.004)$ respectively. Notably, the efficacy of adjuvant ovarian ablation appeared to be similar to that of adjuvant chemotherapy or Tamoxifen in women less than 50 years old in the early EBCTCG analyses [37]. Ovarian ablation had less impact in women who also received chemotherapy, perhaps due either to the influence of chemotherapy-induced amenorrhea, or due to detrimental interactions between concurrent endocrine therapy and chemotherapy. These are not firm conclusions since they are based on indirect comparisons; incomplete data on ER and PR status were available, and age was used as a surrogate determinant of ovarian function. Moreover, these studies generally did not include 
Tamoxifen in the chemotherapy arms, nor did they utilize newer chemotherapy regimens containing anthracyclines and taxanes.

\section{Ovarian Ablation/Ovarian Suppression Compared to Chemotherapy}

The activity of ovarian ablation suggested by the meta-analyses of the EBCTCG led to multiple clinical trials directly comparing the efficacy of ovarian ablation or ovarian suppression to chemotherapy. Eligibility for these trials was determined by actual menopausal status rather than age; for some of these trials, the hormone receptor status of the tumor was also available. These studies are summarized in Table 2.

The Scottish/Imperial Cancer Research Fund trial randomized women to ovarian ablation or $\mathrm{CMF}$, each with or without 5 years of prednisolone [38]. Follow up at 12 years revealed no difference in event-free survival or OS. Subgroup analysis showed that ovarian ablation improved survival for the 270 women whose tumors expressed ER, whereas CMF improved survival for those women whose tumors did not. A Scandinavian trial compared radiationinduced ovarian failure with nine cycles of intravenous CMF [39]. There was no difference between these treatments, with a hazard ratio of ovarian ablation compared to CMF for DFS of 0.99 at 8.5 years median follow up, and for OS of 1.11 at 10.5 years median follow up. The Takeda Adjuvant Breast Cancer Study with Leuprorelin Acetate (TABLE) study randomized 589 patients with lymph node-positive, ER+ breast cancer to receive six cycles of CMF or 2 years of monthly depot Leuprorelin acetate [40]. No differences in recurrencefree survival or OS emerged. The Zoladex Early Breast Cancer Research Association (ZEBRA) study randomized 1,640 premenopausal women with lymph node-positive breast cancer (80\% with ER+ tumors) to six cycles of oral CMF or 2 years of monthly ovarian suppression with goserelin [41]. DFS and OS were equivalent at 6 years median follow up in patients with ER+ disease. Women who developed CMF-related ovarian failure had longer DFS than those who did not; moreover, women with ER- tumors who were treated with CMF had longer DFS than similar women treated with goserelin. Importantly, $76.9 \%$ of women treated with CMF remained amenorrheic at 3 years, where as only $22.6 \%$ of women treated with goserelin remained amenorrheic at this same time point. Together, these trials support the use of goserelin as a reasonable treatment option for young women with ER+, lymph node-positive breast cancer, particularly those who wish to preserve fertility.

\section{Ovarian Suppression Combined with Chemotherapy}

Younger women are less likely to develop permanent amenorrhea as the result of adjuvant chemotherapy [42]. It has been suggested that these women may be at higher risk of relapse than older premenopausal patients with a higher likelihood of chemotherapy-induced amenorrhea, arguing that ovarian ablation/suppression could be an effective adjuvant therapy for them [43]. At least four studies have examined whether the addition of ovarian ablation to standard adjuvant chemotherapy improves outcomes in young women with early breast cancer (Table 2). The Intergroup study INT0101 enrolled 1,503 eligible premenopausal women with lymph node-positive, ER+ breast cancer, randomizing them to six cycles of oral CAF alone (CAF), six cycles of CAF followed by goserelin (CAF-Z), or six cycles of CAF followed by goserelin and tamoxifen (CAF-ZT) [44]. At a median follow up of 9.6 years, treatment with CAF-ZT improved time to recurrence (TTR) and DFS, but not OS (hazard ratios of $0.73,0.74$, and 0.91 with $p$ values of $<0.01,<0.01$, and 0.23 respectively). There was no advantage for the addition of goserelin to CAF (CAF-Z). These data are limited by the lack of a treatment arm evaluating the efficacy of CAF and tamoxifen alone, as tamoxifen was not regarded as an active agent for premenopausal women when INT0101 was launched. An unplanned subset analysis suggested that any benefit associated with CAF-Z was limited to patients younger than 40 years old. The Zoladex in 
Premenopausal Patients (ZIPP) trial used a two by two factorial design in a four arm study comparing 2 years of treatment with tamoxifen, goserelin, tamoxifen and goserelin, or no hormonal therapy [45]. This patient population was heterogeneous: $42 \%$ had positive lymph nodes, $56 \%$ had ER+ breast cancer, and $43 \%$ received adjuvant chemotherapy. At a median follow-up of 5.5 years, goserelin treatment was associated with a $20 \%$ decrease in first events $(\mathrm{p}=0.002)$, and a $19 \%$ improvement in $\mathrm{OS}(\mathrm{p}=0.038)$. The clinical benefit was greatest in patients with ER+ tumors, and was less pronounced in those who also received tamoxifen or chemotherapy. The IBCSG Trial 11-93 treated 174 patients, comparing the efficacy of ovarian ablation/suppression combined with tamoxifen and four cycles of anthracycline-based chemotherapy to that of ovarian ablation/suppression combined with tamoxifen alone in premenopausal women with lymph node-positive, ER+ breast cancer [46]. Although the power of the study is small, no differences emerged. The IBCSG VIII trial compared oral CMF followed by 1.5 years of goserelin to either six cycles of oral CMF alone or 2 years of goserelin alone in 1,063 pre- and perimenopausal women with lymph node-negative breast cancer [47]. The majority of these women had ER+ breast cancers. At a median follow up of 5.7 years, CMF was superior to goserelin in patients with ERdisease, but equivalent to goserelin in ER+ patients. Another trial randomized 926 premenopausal patients with positive lymph nodes or high grade breast cancers to adjuvant chemotherapy alone, or adjuvant chemotherapy plus ovarian ablation/suppression (radiation or triptorelin for 3 years) [48]. In this study, 63\% of enrolled patients had HR+ tumors, and $77 \%$ received anthracycline-based chemotherapy. There was no difference between the treatments arms with regard to 10 year DFS or OS. However, patients younger than 40 years old had a lower rate of recurrence with the addition of ovarian ablation/suppression.

In the aggregate, these trials do not establish a clear benefit for combined ovarian suppression and chemotherapy. However, the subgroup of young premenopausal women less than 40 years of age with ER+ breast cancers who do not become postmenopausal with chemotherapy may benefit from the addition of ovarian ablation. Adequately powered, randomized, prospective clinical trials are essential to define the role of ovarian ablation/ suppression in this patient population.

\section{Ovarian Ablation Combined with Tamoxifen}

Tamoxifen was not routinely used for the management of premenopausal women with hormone-sensitive breast cancer until after the results of the EBCTCG were released in 1995. These data showed that tamoxifen resulted in a proportional reduction in the risk of recurrence and death of $42 \%$ and $32 \%$ respectively, regardless of chemotherapy administration [25]. Two trials have examined the addition of tamoxifen to ovarian ablation in the absence of chemotherapy. An Intergroup trial (INT0142) enrolled 350 premenopausal women with lymph node-negative, hormone receptor-positive breast cancer less than $3 \mathrm{~cm}$ in size, randomizing them to 5 years of tamoxifen or 5 years of tamoxifen plus ovarian ablation. This trial was closed early due to poor accrual, so the data lack statistical power. Nevertheless, there was no difference between treatment with tamoxifen alone or tamoxifen plus ovarian ablation [49]. The second trial conducted in Southeast Asia enrolled 709 women with early breast cancer, randomizing them to receive oophorectomy plus tamoxifen at the time of primary breast surgery or at first relapse [50]. At a mean follow up of 3.6 years, the 5 year DFS rates were $75 \%$ and $58 \%$ respectively ( $\mathrm{p}=0.0075$, adjusted).

Corresponding OS rates were $78 \%$ and $70 \%$ ( $\mathrm{p}=0.41$, unadjusted). The cost efficacy analysis showed a cost per year of life gained of $\$ 351$, compared to $\$ 11,300$ for chemotherapy in the setting of negative lymph nodes, and \$5,000 in the setting of positive lymph nodes. These data support oophorectomy and tamoxifen as active and cost-effective first-line adjuvant therapy for premenopausal women with operable hormone-sensitive breast cancer. 
Several clinical trials have tested the combination of tamoxifen and ovarian ablation/ suppression compared to chemotherapy. The Gruppo di Ricerca in Oncologia Clinica e Terapie Associate (GROCTA) trial compared six cycles of oral CMF to tamoxifen combined with ovarian ablation (surgery, radiation, or 2 years of goserelin) [51]. At a median follow up of 76 months, there was no difference between the treatments. The French Adjuvant Study Group (FASG) 06 trial compared 3 years of tamoxifen plus the LHRH agonist triptoreline to six cycles of FEC in 333 premenopausal women with lymph nodepositive, HR+ breast cancer. At a median follow up of 54 months, there were no significant differences [52]. A small French study compared FAC to ovarian ablation and tamoxifen in women with lymph node-positive, hormone receptor-positive disease [53]. This study similarly showed no difference, although it was underpowered. The Adjuvant Breast Cancer Trialists' Collaborative Group conducted the Ovarian Ablation/Suppression Trial (ABC OAS Trial), enrolling 2,144 pre- and perimenopausal patients receiving 5 years of tamoxifen with or without chemotherapy to receive ovarian ablation/suppression, or no additional therapy. Of these patients, 942 (88\%) received ovarian ablation/suppression. Relapse-free survival (RFS) and OS were similar between the two groups, arguing against the addition of ovarian manipulation to standard adjuvant therapy [54]. Finally, the ABCSG conducted a trial of therapy with 3 years of goserelin plus 5 years of tamoxifen to six cycles of intravenous CMF in 1,045 women with Stage I or II hormone receptor-positive breast cancer [55]. At a mean follow up of 42 months, combination endocrine therapy resulted in a statistically significant improvement in DFS compared to CMF ( $\mathrm{p}=0.02)$, with no difference in OS. Notably, the women in the CMF group who developed ovarian failure had significantly longer DFS and OS compared to those who did not. A limitation of all of these trials is that they failed to incorporate tamoxifen into the chemotherapy arms.

\section{Emerging Data Regarding Endocrine Therapy for Premenopausal Women}

The role of ovarian ablation in the management of premenopausal women with early breast cancer remains uncertain. Recently, the EBCTCG reported a meta-analysis of 11,906 premenopausal women with early breast cancer randomized in 16 trials of LHRH agonists [56]. While the use of LHRH agonists alone was ineffective therapy for hormone receptorpositive tumors, the addition of LHRH agonists to tamoxifen, chemotherapy, or both reduced recurrence by $12.7 \%(\mathrm{p}=0.02)$, and death after recurrence by $15.1 \%(\mathrm{p}=0.03)$. LHRH agonists showed a similar efficacy to chemotherapy, but no trials assessed an LHRH agonist compared to chemotherapy, each followed by tamoxifen. LHRH agonists were ineffective in hormone receptor-negative tumors. Several randomized Phase III clinical trials are prospectively evaluating the role of ovarian ablation/suppression with tamoxifen or an aromatase inhibitor as a component of primary therapy for ER+ breast cancer (Table 3). Additionally, the Oncotype Dx test, a 21-gene risk stratification tool, has recently emerged as a means of determining recurrence risk and guiding therapeutic decision-making [23, 24]. In retrospective studies, women with $\mathrm{ER}+$, lymph node-negative tumors and a high recurrence score appear to benefit from the addition of chemotherapy to endocrine therapy to decrease the risk of relapse, whereas those with low to intermediate recurrence scores benefit primarily from endocrine therapy. A large, prospective trial (TAILORx) has been launched in the United States to validate Oncotype Dx as a tool that can be used to tailor adjuvant therapy to the biology of the primary breast tumor [57]. Finally, for hormone receptor-positive tumors, it is clear that over $50 \%$ of recurrences and over $66 \%$ of breast cancer deaths occur more than 5 years after diagnosis [10, 25, 58]. This observation, along with the results of the MA.17 trial discussed below, supports the concept of extending endocrine treatment beyond 5 years, which is the current standard. Currently, the Adjuvant Tamoxifen: Longer Against Shorter (ATLAS) trial, and the Adjuvant Tamoxifen Treatment, Offer More? (ATTOM) trials are following women treated with Tamoxifen for up to 10 years [59]. The ATLAS study is comparing 5-10 years of adjuvant Tamoxifen therapy, and 
the ATTOM trial is randomizing women who discontinue Tamoxifen treatment after at least 2 years to receive an additional 5 years of Tamoxifen or no additional therapy. In the interim, 5 years of tamoxifen is standard for premenopausal women with hormone receptorpositive breast cancer.

\section{Adjuvant Endocrine Therapy in Postmenopausal Women}

As discussed above, for the last 30 years tamoxifen was the drug of choice for postmenopausal women with hormone receptor positive-breast cancer. It cuts the risk of both disease recurrence and new contralateral breast cancers by about $50 \%$, and reduces mortality by about $31 \%[10,25]$. However, tamoxifen therapy is discontinued by $23-40 \%$ of patients who take it because of issues related to quality of life. Risks of tamoxifen therapy include hot flashes, vaginal bleeding and discharge, endometrial cancer, hysterectomy, ischemic cerebrovascular events, and venous thromboembolic events. Multiple third generation aromatase inhibitors have been developed as an alternative to tamoxifen for postmenopausal women. Of these drugs, anastrozole, letrozole, and exemestane are currently available in the United States.

\section{Aromatase Inhibitors Instead of Tamoxifen}

Several studies have examined the activity of initial adjuvant treatment with aromatase inhibitors (Table 4). The Arimidex, Tamoxifen, Alone or in Combination (ATAC) trial compared 5 years of therapy with anastrozole alone, tamoxifen alone, or the combination of anastrozole and tamoxifen in 9,366 postmenopausal women with hormone receptor-positive early breast cancer [60]. The primary endpoints of this study were DFS and safety/ tolerability. At first analysis, with a median follow-up of 33 months, treatment with anastrozole resulted in a better DFS than treatment with tamoxifen ( 89.4 vs $87.4 \%$ respectively; $\mathrm{p}=0.13$ ). Results for the combination arm were not significantly better than the tamoxifen alone arm, so it was closed. Notably, treatment with anastrozole also reduced the incidence of contralateral breast cancers and distant metastases by 42 and $14 \%$ respectively as compared to tamoxifen. Anastrozole was better tolerated with regard to hot flashes, thromboembolic events, endometrial cancer, vaginal bleeding, and cerebrovascular events, but resulted in a higher rate of musculoskeletal symptoms, bone density loss, and bone fractures. The ATAC trial was recently updated at 68 months of follow up; at this time only $8 \%$ of patients remained on therapy [61]. The DFS for anastrozole compared with tamoxifen was superior, with a hazard ratio of $0.87(\mathrm{p}=0.002)$, and an absolute difference of $3.7 \%$ between the two arms. There is no statistically significant OS difference between the two arms. Based on these data, the U.S. Food and Drug Administration (FDA) approved anastrozole as initial adjuvant endocrine therapy for postmenopausal women with ER+ early breast cancer.

The Breast International Group (BIG) 1-98 trial randomized 8,028 postmenopausal women with hormone receptor-positive breast cancer to 5 years of tamoxifen alone, 5 years of letrozole alone, 2 years of tamoxifen followed by 3 years of letrozole, or 2 years of letrozole followed by 3 years of tamoxifen. The first analysis of this study compared the two groups assigned to initial tamoxifen therapy (4,007 patients) to the two groups assigned to initial letrozole therapy (4,003 patients). At a median follow up of 25.8 months, letrozole significantly improved DFS compared to tamoxifen, with a hazard ratio of $0.81(\mathrm{p}=0.003)$ [62]. Five-year DFS estimates were $84 \%$ for the letrozole group and $81.4 \%$ for the tamoxifen group, for an absolute difference of $2.6 \%$ between the two groups. Letrozole therapy was associated with decreased rates of distant recurrence, with a hazard ratio of 0.73 $(\mathrm{p}=0.001)$. A subsequent update at a median follow up of 51 months revealed an $18 \%$ reduction in the risk of recurrence, with a hazard ratio of $0.82(\mathrm{p}=0.007)$ [63]. These results 
are similar to the results of the ATAC study; the spectrum of side effects for aromatase inhibition and tamoxifen were also similar to those observed in the ATAC trial. There is currently no significant difference in OS between these two groups. Based on these data, letrozole was approved by the U.S. FDA for the adjuvant treatment of postmenopausal women with early ER+ breast cancer. Data from the sequential arms are not yet available.

The Tamoxifen and Exemestane Adjuvant Multicenter (TEAM) trial was designed to randomize 4,400 postmenopausal patients with early breast cancer to exemestane or tamoxifen for 5 years [64]. Based on the results of the Intergroup Exemestane Study (IES) showing that Exemestane given after 2-3 years of Tamoxifen improves DFS compared to tamoxifen alone (discussed below), the TEAM trial has been amended to permit those initially randomized to receive tamoxifen to cross over to exemestane.

\section{Aromatase Inhibitors After Tamoxifen}

Several randomized trials have further analyzed the sequential use of tamoxifen and aromatase inhibitors (Table 5). The Italian ITA study randomized 426 postmenopausal women with hormone receptor-positive breast cancer who had already completed 2 years of tamoxifen to continue tamoxifen to complete a total of 5 years, or to switch to anastrozole for 3 years to complete a total of 5 years of endocrine therapy [65]. At a median follow up of 64 months, sequential therapy with tamoxifen and anastrozole resulted in improved eventfree and RFS, with hazard ratios of $0.57(\mathrm{p}=0.005)$ and $0.56(\mathrm{p}=0.01)$ respectively. There was a trend toward fewer deaths in the anastrozole group, although this did not reach statistical significance. The Austrian Breast and Colorectal Cancer Study Group (ABSCG)-8 and the Arimidex-Nolvadex (ARNO) trials randomized a combined total of 3,224 postmenopausal women who had been on tamoxifen for 2 years to continue tamoxifen to complete a total of 5 years of treatment, or to switch to anastrozole for 3 years to complete a total of 5 years of endocrine therapy [66]. At a median follow up of 28 months, sequential therapy with tamoxifen and anastrozole resulted in a $41 \%$ proportional improvement in DFS $(\mathrm{p}=0.0009)$. At this time, there was a 3.1\% absolute improvement in event-free survival. An updated analysis revealed that, at a median follow up of 30.1 months, there was a $39 \%$ reduction in the relative risk of disease recurrence or death, with a hazard ratio of 0.61 $(p=0.01)$ [67]. It also revealed a survival benefit for sequential therapy, with 15 deaths in the group treated with tamoxifen followed by anastrozole compared to 28 deaths in the group treated with tamoxifen alone $(\mathrm{p}=0.045)$. The International Exemestane Study (IES) randomized 4,742 postmenopausal women with hormone receptor-positive breast cancer who had already received 2-3 years of adjuvant tamoxifen to complete 5 years total of tamoxifen, or switch to exemestane to complete a total of 5 years of endocrine therapy [68]. At a median follow up of 36 months, sequential treatment with tamoxifen and exemestane resulted in proportional and absolute reductions in the risk of recurrence of 32 and $4.7 \%$ respectively $(\mathrm{p}=0.0005)$. Subsequent analysis of these data showed that the DFS benefit was maintained, with a hazard ratio of $0.76(\mathrm{p}=0.0001)$, and a statistically significant survival benefit was associated with sequential therapy in the ER+/ER unknown subgroup of patients. At the most recent report of these results with a median follow up of 55.7 months, there was a $24 \%$ improvement in DFS with exemestane therapy, with a hazard ratio of 0.76 $(\mathrm{p}=0.0001)$, and an absolute benefit of $3.3 \%$ [69]. Further, there was a $15 \%$ improvement in OS in this group, with a hazard ratio of $0.85(\mathrm{p}=0.08)$. It is important to remember that only those patients who had completed 2-3 years of tamoxifen without early recurrence were included in this study, so it is a highly selected patient population compared to those populations treated initially with endocrine therapy in the ATAC or BIG 1-98 studies.

Finally, the MA.17 trial randomized 5,187 women to 5 years of letrozole or placebo after completing 5 years of adjuvant tamoxifen therapy [70,71]. At a median follow up of 30 
months, letrozole was associated with a statistically significantly better DFS and distant DFS than placebo, with HRs of $0.58(\mathrm{p}<0.001)$ and $0.60(\mathrm{p}=0.002)$ respectively. There was no difference between the arms for OS. However, the OS of lymph node-positive patients was significantly improved with letrozole, with a HR of $0.61(\mathrm{p}=0.04)$.

The data were unblinded in October 2003, and all patients were offered open-label letrozole. Among the 2,594 women initially randomized to placebo, 1,655 chose to receive open-label letrozole. As a group, these women were younger, had disease at higher risk for relapse, and were more likely to have been treated with chemotherapy than those who did not choose to take letrozole. At 54 months of follow up, there was a statistically significant improvement in DFS in women who chose to take letrozole, with a hazard ratio of $0.31(\mathrm{p}<0.0001)$ [72]. Thus, women with hormone-sensitive breast cancer may benefit from letrozole therapy even after a significant period of time off tamoxifen therapy. Based on the results of the MA.17 study, the U.S. FDA approved letrozole as extended adjuvant endocrine therapy after 5 years of tamoxifen.

A retrospective analysis of this study demonstrated that the impact of letrozole compared to placebo may be greatest in women with ER+/PR+ tumors [73]. Furthermore, data from cohorts of patients defined by randomization date $(12,24,36$, and 48 months) revealed a significant decrease in the risk of recurrence with letrozole treatment regardless of time from randomization [74]. The hazard ratios for DFS continued to decrease over time, from 0.52 at 12 months to 0.19 at 48 months. This observation suggests that longer letrozole exposure affords greater clinical benefit, at least out to 48 months. The MA.17R trial is an extension of the MA.17 trial that randomly assigns patients to either five more years of letrozole, or to observation. This study will provide data guiding the optimal duration of treatment for efficacy in addition to long-term toxicity for patients treated over 10 years from initial diagnosis.

No direct comparisons of the aromatase inhibitors in the adjuvant setting have been reported. MA. 27 is a randomized, Phase III trial comparing 5 years of anastrozole to 5 years of exemestane as initial therapy for patients with early hormone receptor-positive breast cancer. The Femara vs Anastrozole Clinical Evaluation (FACE) trial is a randomized Phase III study comparing the efficacy and safety of letrozole and anastrozole in postmenopausal patients with lymph node-positive early breast cancer.

A small trial conducted by the Austrian Breast and Colorectal Cancer Study Group (ABCSG), trial ABCSG 6a, examined the addition of 3 years of adjuvant anastrozole therapy after the completion of 5 years of adjuvant tamoxifen therapy [75]. There was longer event-free survival in women who received sequential therapy compared to 5 years of tamoxifen alone with or without aminoglutethimide. Finally, a meta-analysis of the "switching trials", with 4,006 patients and a median follow up of 30 months, confirmed an improvement in DFS, with a hazard ratio of $0.59(\mathrm{p}=0.0001)$ [76]. The hazard ratio for OS was $0.71(\mathrm{p}=0.038)$.

All of the clinical trials examining the efficacy of adjuvant aromatase inhibitors have demonstrated an adverse effect on bone mineral density. A sub-protocol of the ATAC trial studied 308 patients from the study, and a control group of 46 nonrandomized postmenopausal patients with invasive, early breast cancer who did not receive endocrine therapy [77]. Anastrozole was associated with a decrease in bone mineral density in the spine and hip at both 1 and 2 years, whereas tamoxifen was associated with an increase. The rate of bone loss with anastrozole occurred at a constant rate over the 2-year period. The analysis of bone mineral density at 5 years showed that bone loss continued unabated in the hip, but that the rate of bone loss decreased in the lumbar spine ( $\mathrm{p}=0.0002)$. Importantly, no 
patient with a normal baseline bone density developed osteoporosis by 5 years. A similar analysis was performed on the IES study [78]. Within 6 months of switching to exemestane, baseline bone mineral density was lowered by $2.7 \%(\mathrm{p}<0.0001)$ at the lumbar spine and $1.4 \%(\mathrm{p}<0.0001)$ at the hip. Bone mineral density decreases were only $1.0 \%(\mathrm{p}=0.002)$ and $0.8 \%(\mathrm{p}=0.003)$ in year 2 at the lumbar spine and hip, respectively. No patient with normal bone mineral density at trial entry developed osteoporosis. The NCIC CTC MA.17 study also reported similar findings [79]; 226 patients (122 letrozole, 104 placebo) were enrolled. At 24 months, patients receiving letrozole had a significant decrease in total hip and lumbar spine bone mineral density compared to those receiving placebo, with a loss of $3.6 \%$ compared to $0.71 \%(\mathrm{p}=0.044)$ and $5.35 \%$ compared to $0.70 \%(\mathrm{p}=0.008)$ respectively. No patient developed osteoporosis in the hip, whereas at the L2- L4 (posteroanterior view), more women developed osteoporosis while receiving letrozole compared to those receiving placebo, at 4.1 and $0 \%$ respectively $(\mathrm{p}=0.064)$. Other side effects commonly reported with the use of aromatase inhibitors are hot flashes and arthralgias. Aromatase inhibitors may also adversely impact the lipid profile, potentially resulting in a slight increase in cardiovascular disease; this is under active investigation.

\section{Recommendations for Adjuvant Endocrine Therapy from Consensus Meetings}

Several consensus statements about the use of adjuvant endocrine therapy have been released. In 2000, the United States NIH Consensus Development Conference on the Adjuvant Therapy for Breast Cancer concluded that standard adjuvant chemotherapy should be recommended for the majority of premenopausal women with early stage breast cancer. Women with ER+ breast cancer should be given tamoxifen for 5 years, with consideration of ovarian ablation/suppression as an alternative to tamoxifen for selected women. The panel concluded that there was insufficient evidence to support the addition of ovarian ablation/ suppression to standard adjuvant chemotherapy combined with tamoxifen [80]. The American Society of Clinical Oncology convened a Technology Assessment Panel in 2002, 2003, and 2004 to review data on the use of aromatase inhibitors in the adjuvant setting. The panel concluded that the optimal adjuvant endocrine therapy for postmenopausal women with hormone sensitive breast cancer should include an aromatase inhibitor as initial therapy, or after a period of tamoxifen therapy [81].

The 2005 International Consensus Conference on the Adjuvant Therapy of Primary Breast Cancer emphasized that the first consideration for adjuvant breast cancer therapy should be the endocrine responsiveness of the tumor [22]. The Conference defined breast cancers as endocrine-responsive, endocrine-nonresponsive, and tumors of uncertain endocrine responsiveness, with each category further divided by menopausal status. Importantly, axillary lymph node involvement itself did not define high risk. Intermediate risk tumors included lymph node-negative disease if the primary tumor presented high risk features, and lymph node-positive disease (one to three positive lymph nodes) if the tumor did not present high risk features. The panel recommended chemotherapy for endocrine-nonresponsive disease, endocrine therapy for endocrine-responsive disease, and the combination of endocrine therapy and chemotherapy for some intermediate and all high risk disease. Both endocrine therapy and chemotherapy were recommended for tumors of uncertain endocrine responsiveness, except for those tumors in the low risk category (small, lymph nodenegative tumors). 


\section{Lingering Questions, Emerging Considerations and Future Directions for Research}

Despite its longstanding use as the mainstay of therapy for hormone-responsive breast cancer, many issues remain unresolved regarding distinct aspects of endocrine manipulation. For premenopausal women, the benefit of adding ovarian ablation/suppression to those premenopausal women who do not become amenorrheic as the result of adjuvant chemotherapy remains unknown, and ongoing clinical trials are addressing this issue. These trials are also testing the use of combined endocrine therapy with ovarian ablation/ suppression and aromatase inhibition in this setting. The appropriate duration of the use of medical ovarian suppression also remains unknown - most clinical trials have continued LHRH analogues for 2-3 years. For postmenopausal women, the optimal use of aromatase inhibition remains to be defined. The most appropriate sequencing strategy with tamoxifen, the optimal duration of therapy, and the optimal aromatase inhibitor remain unknown. It is critical to remember that aromatase inhibitors are contraindicated in women who are premenopausal, and should be used with extreme caution in women with chemotherapyinduced amenorrhea [82]. For both premenopausal and postmenopausal women, the longterm sequelae of estrogen deprivation with ovarian ablation/suppression and/or aromatase inhibition are also unknown. Finally, host and tumor biology are becoming increasingly important in therapeutic decision-making. Pharmacogenomic variables impacting the efficacy of endocrine therapy have emerged as important considerations in choosing tamoxifen therapy, and molecular determinants of tumor biology distinct from the endocrine signaling pathways have been suggested as potential predictors of improved response to therapy with aromatase inhibitors as compared to tamoxifen.

\section{References}

1. Osborne CK, Yochmowitz MG, Knight WA III, McGuire WL. The value of estrogen and progesterone receptors in the treatment of breast cancer. Cancer. 1980; 46(12 Suppl):2884-8. [PubMed: 7448733]

2. Harvey J, Clark G, Osborne C, Allred D. Estrogen receptor status by immunohistochemistry is superior to the ligand-binding assay for predicting response to adjuvant endocrine therapy in breast cancer. J Clin Oncol. 1999; 17:1474-81. [PubMed: 10334533]

3. Mohsin S, Wiess H, Gutierrez M, et al. Neoadjuvant Trastuzumab induces apoptosis in primary breast cancers. J Clin Oncol. 2005; 23:2460-8. [PubMed: 15710948]

4. Goldhirsch A, Wood W, Gelber R, et al. Meeting highlights: Updated international expert consensus on the primary therapy of early breast cancer. J Clin Oncol. 2003; 21:3357-65. [PubMed: 12847142]

5. Zafrani B, Aubriot H, Mouret E, et al. High sensitivity and specificity of immunohistochemistry for the detection of hormone receptors in breast carcinoma: Comparison with biochemical determination in a prospective study of 793 cases. Histopathol. 2000; 37:536-45.

6. Clarke M. Ovarian ablation in breast cancer, 1896-1998: Milestones along hierarchy of evidence from case report to Cochrane review. BM J. 1998; 317:1246-8.

7. Smith IE, Dowsett M. Aromatase inhibitors in breast cancer. N Engl J Med. 2003; 348:2431-42. [PubMed: 12802030]

8. Osborne CK, Zhao H, Fuqua SA. Selective estrogen receptor modulators: Structure, function, and clinical use. J Clin Oncol. 2000; 18(17):3172-86. [PubMed: 10963646]

9. Dauvois S, Danielian PS, White R, Parker MG. Antiestrogen ICI 164,384 reduces cellular estrogen receptor content by increasing its turnover. Proc Natl Acad Sci U S A. 1992; 89(9):4037-41. [PubMed: 1570330]

10. Early Breast Cancer Trialists' Collaborative Group (EBCTCG). Effects of chemotherapy and hormonal therapy for early breast cancer on recurrence and 15-year survival: An overview of the randomized trials. Lancet. 2005; 365:1687-717. [PubMed: 15894097] 
11. Perou C, Sorlie T, Eisen M, et al. Molecular portraits of human breast tumors. Nature. 2000; 406:747-52. [PubMed: 10963602]

12. Sorlie T, Perou C, Tibshirani R, et al. Gene expression patterns of breast carcinomas distinguish tumor subclasses with clinical implications. Proc Natl Acad Sci U S A. 2001; 98(19):10869-74. [PubMed: 11553815]

13. Sorlie T, Tibshirani R, Parker J, et al. Repeated observation of breast tumor subtypes in independent gene expression data sets. Proc Natl Acad Sci U S A. 2003; 100(14):8418-23. [PubMed: 12829800]

14. Dowsett M, Harper-Wynne C, Beoddinghaus I, et al. HER-2 amplification impedes the antiproliferative effects of hormone therapy in estrogen receptor-positive primary breast cancer. Cancer Res. 2001; 61:8452-8. [PubMed: 11731427]

15. Moy B, Goss P. Estrogen receptor pathways: resistance to endocrine therapy and new therapeutic approaches. Clin Cancer Res. 2006; 15:4790-3. [PubMed: 16914563]

16. Choi J, Nowell S, Blanco J, Ambrosone C. The role of genetic variability in drug metabolism pathways in breast cancer prognosis. Pharmacogenomics. 2006; 7:613-24. [PubMed: 16753008]

17. Wiebe V, Osborne C, Fuqua S, DeGregorio M. Tamoxifen resistance in breast cancer. Crit Rev Oncol Hematol. 1993; 14:173-88. [PubMed: 8397846]

18. Smigal C, Jemal A, Ward E, et al. Trends in breast cancer by race and ethnicity: Update 2006. CA Cancer J for Clinicians. 2006; 56:168-83.

19. Pujol P, Daures J, Thezenas S, et al. Changing estrogen and progesterone receptor patterns in breast carcinoma during the menstrual cycle and menopause. Cancer. 1998; 83:698-705. [PubMed: 9708933]

20. Goldhirsch A, Glick J, Gelber R, et al. Meeting highlights: International consensus panel on the treatment of primary breast cancer. Seventh international conference on adjuvant therapy of primary breast cancer. J Clin Oncol. 2001; 19(18):3817-27. [PubMed: 11559719]

21. Prowell T, Davidson N. What is the role of ovarian ablation in the management of primary and metastatic breast cancer today? Oncologist. 2004; 9:507-17. [PubMed: 15477635]

22. Goldhirsch A, Glick J, Gelber R, et al. Meeting highlights: International expert consensus on the primary therapy of early breast cancer 2005. Annals Oncol. 2005; 16:1569-83.

23. Paik S, Shak S, Tang G, et al. A multigene assay to predict recurrence of tamoxifen-related, nodenegative breast cancer. N Engl J Med. 2004; 351:2817-26. [PubMed: 15591335]

24. Paik S, Tang G, Shak S, et al. Gene expression and benefit of chemotherapy in women with nodenegative, estrogen-receptor-positive breast cancer. J Clin Oncol. 2006; 24:3726-34. [PubMed: 16720680]

25. Early Breast Cancer Clinical Trialists' Collaborative Group (EBCTCG). Tamoxifen for early breast cancer: Overview of the randomized trials. Lancet. 1998; 351:1451-67. [PubMed: 9605801]

26. Nolvadex Adjuvant Trial Organization (NATO). Controlled trial of tamoxifen as a single adjuvant agent in the management of early breast cancer. Br J Cancer. 1988; 57:608-11. [PubMed: 2900647]

27. Fisher B, Costantino J, Redmond C, et al. A randomized clinical trial evaluating tamoxifen in the treatment of patients with node-negative breast cancer who have estrogen-receptor-positive tumors. N Engl J Med. 1989; 320:479-84. [PubMed: 2644532]

28. Fisher B, Dignam J, Bryant J, Wolmark N. Five versus more than five years of tamoxifen for lymph node-negative breast cancer: Updated findings from the National Surgical Adjuvant Breast and Bowel Project B-14 randomized trial. J Natl Cancer Inst. 2001; 93:684-90. [PubMed: 11333290]

29. Stewart H. The Scottish trial of adjuvant tamoxifen in node-negative breast cancer. J Natl Cancer Inst. 1992; 11:117-20.

30. Stewart H, Forrest A, Everington D, et al. Randomised controlled trial of conservation therapy for breast cancer: 6-year analysis of the Scottish trial. Scottish Cancer Trials Breast Group. Lancet. 1996; 348:708-13. [PubMed: 8806289]

31. Stewart H, Prescott R, Forrest A. Scottish adjuvant tamoxifen trial: A randomized study updated to 15 years. J Natl Cancer Inst. 2001; 93:456-62. [PubMed: 11259471] 
32. Boccardo F, Amoroso D, Rubagotti A, et al. Endocrine therapy of breast cancer: The experience of the Italian cooperative group for chemohormonal therapy of early breast cancer. Annals New York Acad Sci. 1993; 698:318-29.

33. Kaufmann M, Jonat W, Abel U, et al. Adjuvant randomized trials of doxorubicin/ cyclophosphamide versus doxorubicin/cyclophosphamide/tamoxifen and CMF chemotherapy versus tamoxifen in women with node-positive breast cancer. J Clin Oncol. 1993; 11:454-60. [PubMed: 8445420]

34. Tormey DC, Gray R, Abeloff MD, et al. Adjuvant therapy with a doxorubicin regimen and longterm tamoxifen in premenopausal breast cancer patients: An Eastern Cooperative Oncology Group trial. J Clin Oncol. 1992; 10:1848-56. [PubMed: 1453199]

35. Colleoni M, Gelber S, Goldhirsch A, et al. Tamoxifen after adjuvant chemotherapy for premenopausal women with lymph node-positive breast cancer: International Breast Cancer Study Group Trial 13-93. J Clin Oncol. 2006; 24:1332-41. [PubMed: 16505417]

36. Beatson G. On the treatment of inoperable cases of carcinoma of the mamm: Suggestions for new method of treatment. Lancet. 1896; 2:104-7.

37. Early Breast Cancer Trialists' Collaborative Group (EBCTCG). Ovarian ablation in early breast cancer: overview of the randomized trials. Lancet. 1996; 348:1189-96. [PubMed: 8898035]

38. Scottish Cancer Trials Breast Group and ICRF, Breast Unit, Guy's Hospital, London. Adjuvant ovarian ablation versus CMF chemotherapy in premenopausal women with pathological stage II breast carcinoma: the Scottish trial. Lancet. 1993; 341:1293-8. [PubMed: 8098446]

39. Ejlertsen B, Mouridsen H, Jensen M, et al. Similar efficacy for ovarian ablation compared with cylcophosphamide, methotrexate, and fluorouracil: From a randomized comparison of premenopausal patients with node-positive, hormone receptor-positive breast cancer. J Clin Oncol. 2006; 24:4956-62. [PubMed: 17075113]

40. Schmid P, Untch M, Wallwiener D, et al. Cyclophosphamide, methotrexate and fluorouracil (CMF) versus hormonal ablation with leuprorelin acetate as adjuvant treatment of node-positive, premenopausal breast cancer patients: preliminary results of the TABLE-study (Takeda Adjuvant Breast cancer study with Leuprorelin Acetate). Anticancer Res. 2002; 22:2325-32. [PubMed: 12174922]

41. Jonat W, Kaufmann M, Sauerbrei W, et al. Goserelin versus cyclophosphamide, methotrexate, and fluorouracil as adjuvant therapy in premenopausal patients with node-positive breast cancer: The Zoladex Early Breast Cancer Research Association study. J Clin Oncol. 2002; 20:4628-35. [PubMed: 12488406]

42. Petrek J, Naughton M, Case L, et al. Incidence, time course, and determinants of menstrual bleeding after breast cancer treatment: A prospective study. J Clin Oncol. 2006; 24:1045-51. [PubMed: 16476708]

43. Goldhirsch A, Gelber R, Yothers G, et al. Adjuvant therapy for very young women with breast cancer: need for tailored treatments. J Natl Cancer Inst Monogr. 2001; 30:44-51. [PubMed: 11773291]

44. Davidson N, O'Neill A, Vukov A, et al. Chemoendocrine therapy for premenopausal women with axillary lymph node-positive, steroid hormone receptor-positive breast cancer: Results from INT0101 (E5188). J Clin Oncol. 2005; 23:5973-82. [PubMed: 16087950]

45. Baum M, Hackshaw A, Houghton J, et al. Adjuvant goserelin in pre-menopausal patients with early breast cancer: Results from the ZIPP study. Eur J Cancer. 2006; 42:895-904. [PubMed: 16545560]

46. International Breast Cancer Research Study Group. Randomized controlled trial of ovarian function suppression plus tamoxifen versus the same endocrine therapy plus chemotherapy: Is chemotherapy necessary for premenopausal women with node-positive, endocrine-responsive breast cancer? First results of International Breast Cancer Study Group Trial 11-93. Breast. 2001; 10:130-8.

47. Castiglione-Gertsch M, O'Neill A, Price K, et al. Adjuvant chemotherapy followed by goserelin versus either modality alone for premenopausal lymph node-negative breast cancer: A randomized trial. J Natl Cancer Inst. 2003; 95:1833-46. [PubMed: 14679153] 
48. Arriagada R, Le M, Spielmann M, et al. Randomized trial of adjuvant ovarian suppression in 926 premenopausal patients with early breast cancer treated with adjvuant chemotherapy. Annals Oncol. 2005; 16:389-96.

49. Robert N, Wang M, Wood W. Phase III comparison of tamoxifen with ovarian ablation in premenopausal women with axillary node-negative, receptor-positive breast cancer $<3 \mathrm{~cm}$. Proc Am Soc Clin Oncol. 2003; 22:16a.

50. Love R, Duc N, Allred D, et al. Oophorectomy and tamoxifen adjuvant therapy in premenopausal Vietnamese and Chinese women with operable breast cancer. J Clin Oncol. 2002; 20:2559-66. [PubMed: 12011136]

51. Boccardo F, Rubagotti A, Amoroso D, et al. Cyclophosphamide, methotrexate, and fluorouracil versus tamoxifen plus ovarian suppression as adjuvant treatment of estrogen receptor-positive pre-/perimenopausal breast cancer patients: results of the Italian Breast Cancer Adjuvant Study Group 02 randomized trial. J Clin Oncol. 2000; 18:2718-27. [PubMed: 10894871]

52. Roche H, Kerbrat P, Bonneterre J, et al. Complete hormonal blockade versus epirubicin-based chemotherapy in premenopausal, one to three node-positive, and hormone-receptor positive, early breast cancer patients: 7-year follow-up results of French Adjuvant Study Group 06 randomised trial. Annals Oncol. 2006; 17:1221-7.

53. Roche H, Mihura J, de Lafontan B, et al. Castration and tamoxifen versus chemotherapy (FAC) for premenopausal, node- and receptor-positive breast cancer patients: A randomized trial with a 7 years median follow up. Proc Am Soc Clin Oncol. 1996; 15:117.

54. The Adjuvant Breast Cancer Trials Collaborative Group. Ovarian ablation or suppression in premenopausal early breast cancer: Results from the International Adjuvant Breast Cancer Ovarian Ablation or Suppression Randomized Trial. J Natl Cancer Inst. 2007; 99:516-25. [PubMed: 17405996]

55. Jakesz R, Hausmaninger H, Kubista E, et al. Randomized adjuvant trial of tamoxifen and goserelin versus cyclophosphamide, methotrexate, and fluorouracil: Evidence for the superiority of treatment with endocrine blockade in premenopausal patients with hormone- responsive breast cancer-Austrian Breast and Colorectal Cancer Study Group Trial 5. J Clin Oncol. 2002; 20:46217. [PubMed: 12488405]

56. Early Breast Cancer Trialists' Collaborative Group (EBCTCG). Use of luteinising-hormonereleasing hormone agonists as adjuvant treatment in premenopausal patients with hormonereceptor-positive breast cancer: a meta-analysis of individual patient data from randomized adjuvant trials. Lancet. 2007; 369:1711-23. [PubMed: 17512856]

57. Paik S. Molecular profiling of breast cancer. Curr Opin Obst Gynecol. 2006; 18:59-62. [PubMed: 16493262]

58. Kennecke H, Olivotto I, Speers C, et al. Late risk of relapse and mortality among postmenopausal women with estrogen responsive early breast cancer after 5 years of tamoxifen. Annals Oncol. 2007; 18:45-51.

59. Bryant J, Fisher B, Dignam J. Duration of adjuvant tamoxifen therapy. J Natl Cancer Inst Monogr. 2001; 30:56-61. [PubMed: 11773293]

60. Baum M, Budzar A, Cuzick J, et al. Anastrozole alone or in combination with tamoxifen versus tamoxifen alone for adjuvant treatment of postmenopausal women with early breast cancer: First results of the ATAC randomised trial. Lancet. 2002; 359:2131-9. [PubMed: 12090977]

61. Howell A, Cuzick J, Baum M, et al. Results of the ATAC (Arimidex, Tamoxifen, Alone or in Combination) trial after completion of 5 years' adjuvant treatment for breast cancer. Lancet. 2005; 365:60-2. [PubMed: 15639680]

62. Thurlimann B, Keshaviah A, Coates AS, et al. Breast International Group (BIG) 1-98 Collaborative Group: A comparison of letrozole and tamoxifen in postmenopausal women with early breast cancer. N Engl J Med. 2005; 353:2747-57. [PubMed: 16382061]

63. Coates A, Keshaviah A, Thurlimann B, et al. Five years of letrozole compared with tamoxifen as initial adjuvant therapy for postmenopausal women with endocrine-responsive early breast cancer: Update of study BIG 1-98. J Clin Oncol. 2007; 25:486-92. [PubMed: 17200148]

64. Ryan P, Goss P. Adjuvant hormonal therapy in peri- and postmenopausal breast cancer. The Oncologist. 2006; 11:718-31. [PubMed: 16880231] 
65. Boccardo F, Rubagotti A, Puntoni M, et al. Switching to anastrozole versus continued tamoxifen treatment of early breast cancer: preliminary results of the Italian Tamoxifen Anastrozole Trial. J Clin Oncol. 2005; 23:5138-47. [PubMed: 16009955]

66. Jakesz R, Jonat W, Gnant M, et al. Switching of postmenopausal women with endocrineresponsive early breast cancer to anastrozole after 2 years' adjuvant tamoxifen: combined results of ABCSG trial 8 and ARNO 95 trial. Lancet. 2005; 366:455-62. [PubMed: 16084253]

67. Kaufmann M, Jonat W, Hilfrich H, et al. Survival benefit of switching to anastrozole after 2 years of treatment with tamoxifen versus continued tamoxifen therapy: The ARNO 95 study (abstract 547). J Clin Oncol. 2006; 24(18S):14s.

68. Coombes R, Hall E, Gibson L, et al. A randomized trial of exemestane after two to three years of tamoxifen therapy in postmenopausal women with primary breast cancer. N Engl J Med. 2004; 350:1081-92. [PubMed: 15014181]

69. Coombes R, Kilburn L, Snowdon C, et al. Survival and safety of exemestane versus tamoxifen after 2-3 years' tamoxifen treatment (Intergroup Exemestane Study): A randomised controlled trial. Lancet. 2007; 369:559-70. [PubMed: 17307102]

70. Goss P, Ingle J, Martino S, et al. A randomized trial of letrozole in postmenopausal women after five years of tamoxifen therapy for early-stage breast cancer. N Engl J Med. 2003; 349:1793-802. [PubMed: 14551341]

71. Goss P, Ingle J, Martino S, et al. Randomized trial of letrozole following tamoxifen as extended adjuvant therapy in receptor-positive breast cancer: updated findings from NCIC CTG MA.17. J Natl Cancer Inst. 2005; 97:1262-71. [PubMed: 16145047]

72. Robert N, Goss P, Ingle J, et al. Updated analysis of NCIC CTG MA.17 (letrozole vs placebo to letrozole vs placebo) post unblinding (abstract 550). Proc Am Soc Clin Oncol. 2006; 24(18s):5s.

73. Goss P, Ingle J, Martino S, et al. Efficacy of letrozole extended adjuvant therapy according to estrogen receptor and progesterone receptor status of the primary tumor: National Cancer Institute of Canada Clinical Trials Group MA.17. J Clin Oncol. 2007; 25:2006-11. [PubMed: 17452676]

74. Ingle J, Tu D, Pater J, et al. Duration of letrozole treatment and outcomes in the placebo-controlled NCIC CTG MA.17 extended adjuvant therapy trial. Breast Cancer Res Treat. 2006; 99:295-300. [PubMed: 16541302]

75. Jakesz R, Samonigg R, Greil M, et al. Extended adjuvant treatment with anastrozole: Results from the Austrian Breast and Colorectal Cancer Study Group Trial 6a (ABCSG-6a). Proc Am Soc Clin Oncol. 2005; 23(16s):10s.

76. Jonat W, Gnant M, Boccardo F, et al. Effectiveness of switching from adjuvant tamoxifen to anastrozole in postmenopausal women with hormone-sensitive early-stage breast cancer: A metaanalysis. Lancet Oncol. 2006; 7:991-6. [PubMed: 17138220]

77. Eastell R, Hannon RA, Cuzick J, et al. Effect of an aromatase inhibitor on bone mineral density and bone turnover markers: 2-year results of the Anastrozole, Tamoxifen, Alone or in Combination (ATAC) trial (18233230). J Bone Mineral Res. 2006; 21:1215-23.

78. Coleman R, Banks L, Girgis S, et al. Skeletal effects of exemestane on bone-mineral density, bone biomarkers, and fracture incidence in postmenopausal women with early breast cancer participating in the Intergroup Exemestane Study (IES): A randomised controlled study. Lancet Oncol. 2007; 8:119-27. [PubMed: 17267326]

79. Perez EA, Josse RG, Pritchard KI, et al. Effect of letrozole versus placebo on bone mineral density in women with primary breast cancer completing 5 or more years of adjuvant tamoxifen: A companion study to NCIC CTG MA.17. J Clin Oncol. 2006; 24:3629-35. [PubMed: 16822845]

80. National Institutes of Health Consensus Development Panel. National Institutes of Health Consensus Development Conference Statement: adjuvant therapy for breast cancer, November 13, 2000. J Natl Cancer Inst Monogr. 2001; 30:5-15. [PubMed: 11773285]

81. Winer E, Hudis C, Burstein H, et al. American Society of Clinical Oncology technology assessment on the use of aromatase inhibitors as adjuvant therapy for postmenopausal women with hormone receptor-positive breast cancer: Status report 2004. J Clin Oncol. 2005; 23:619-29.

[PubMed: 15545664] 
82. Smith I, Dowsett M, Yap Y, et al. Adjuvant aromatase inhibitors for early breast cancer after chemotherapy-induced amenorrhoea: Caution and suggested guidelines. J Clin Oncol. 2006; 24:2444-7. [PubMed: 16735701] 


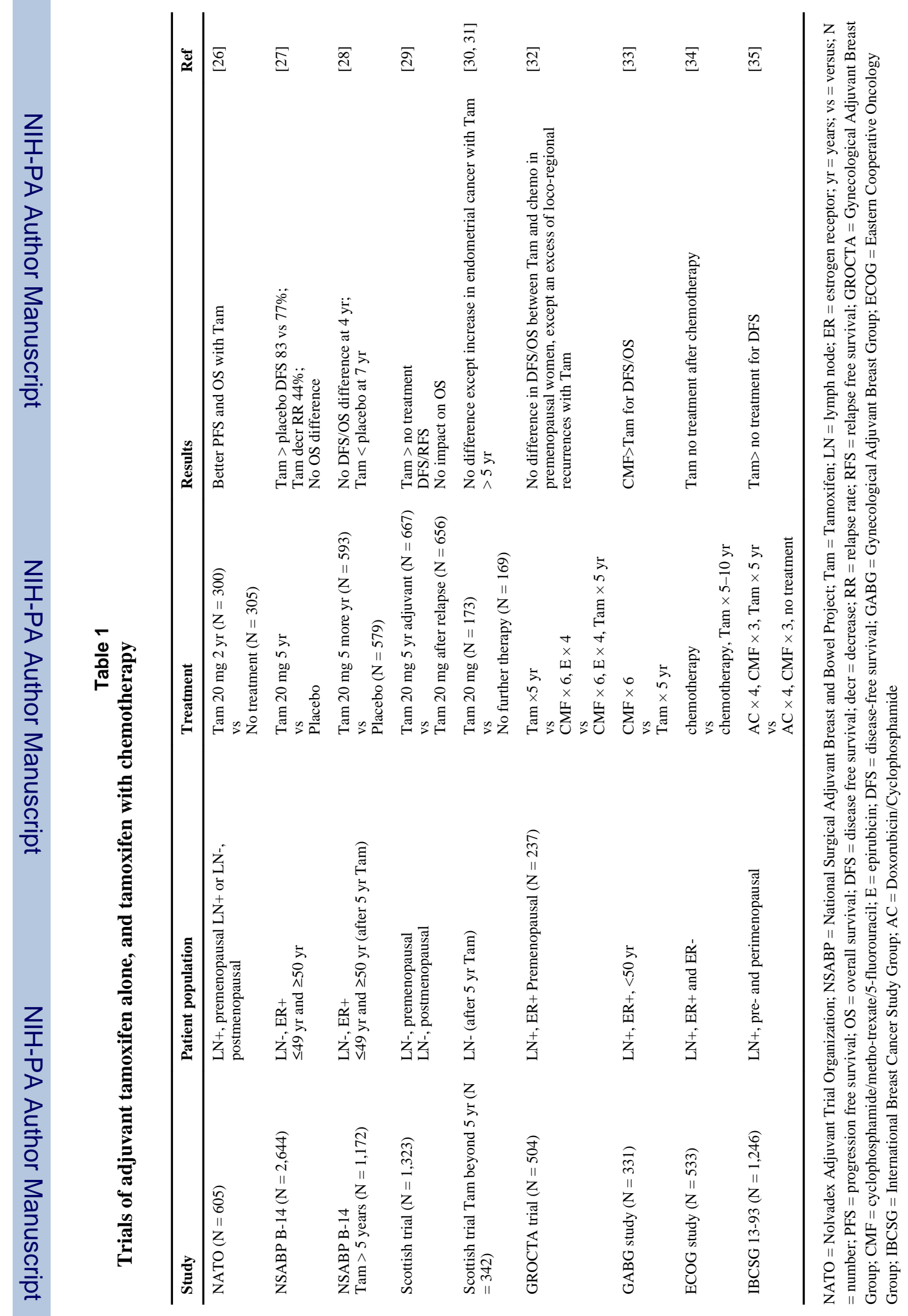




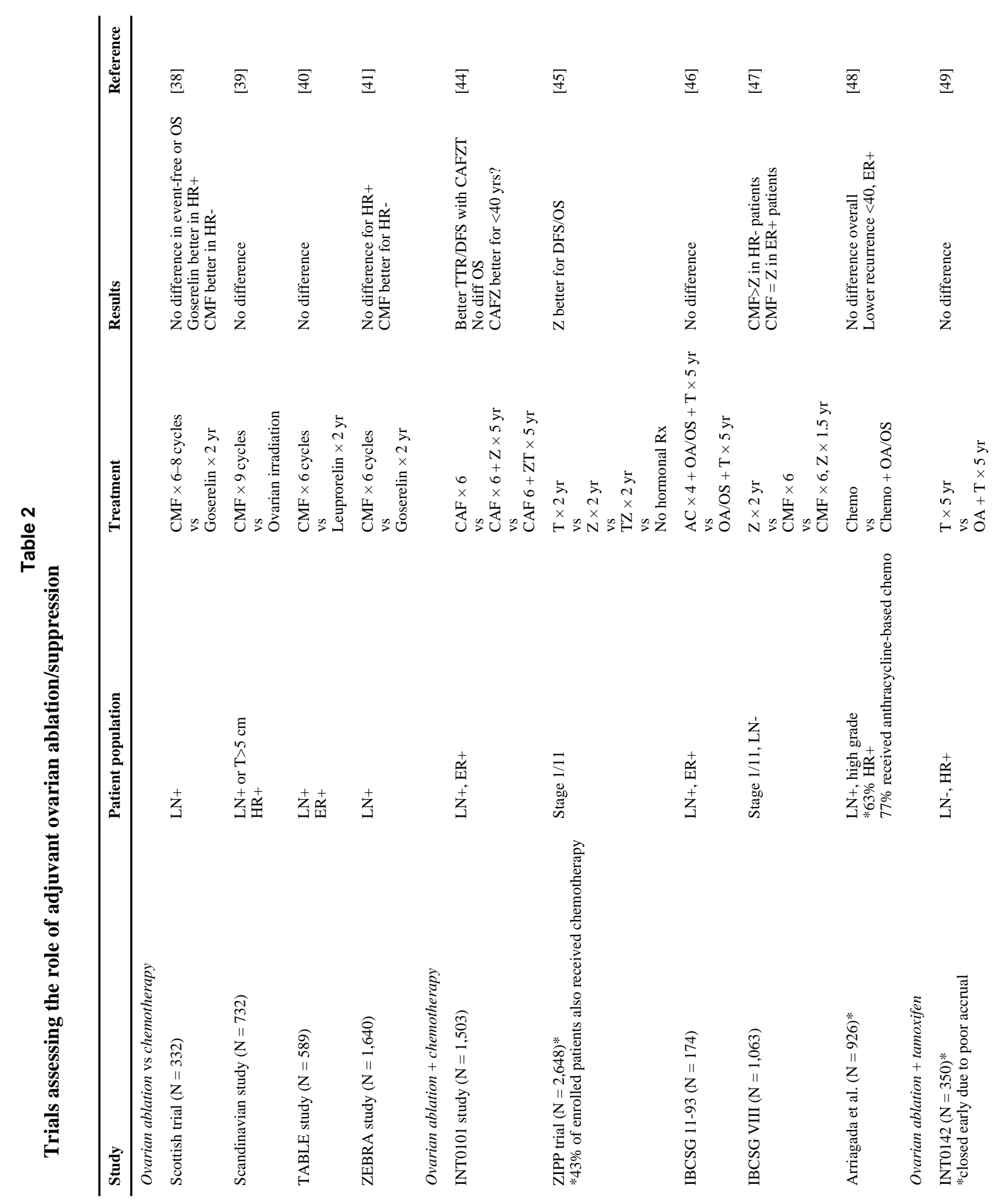




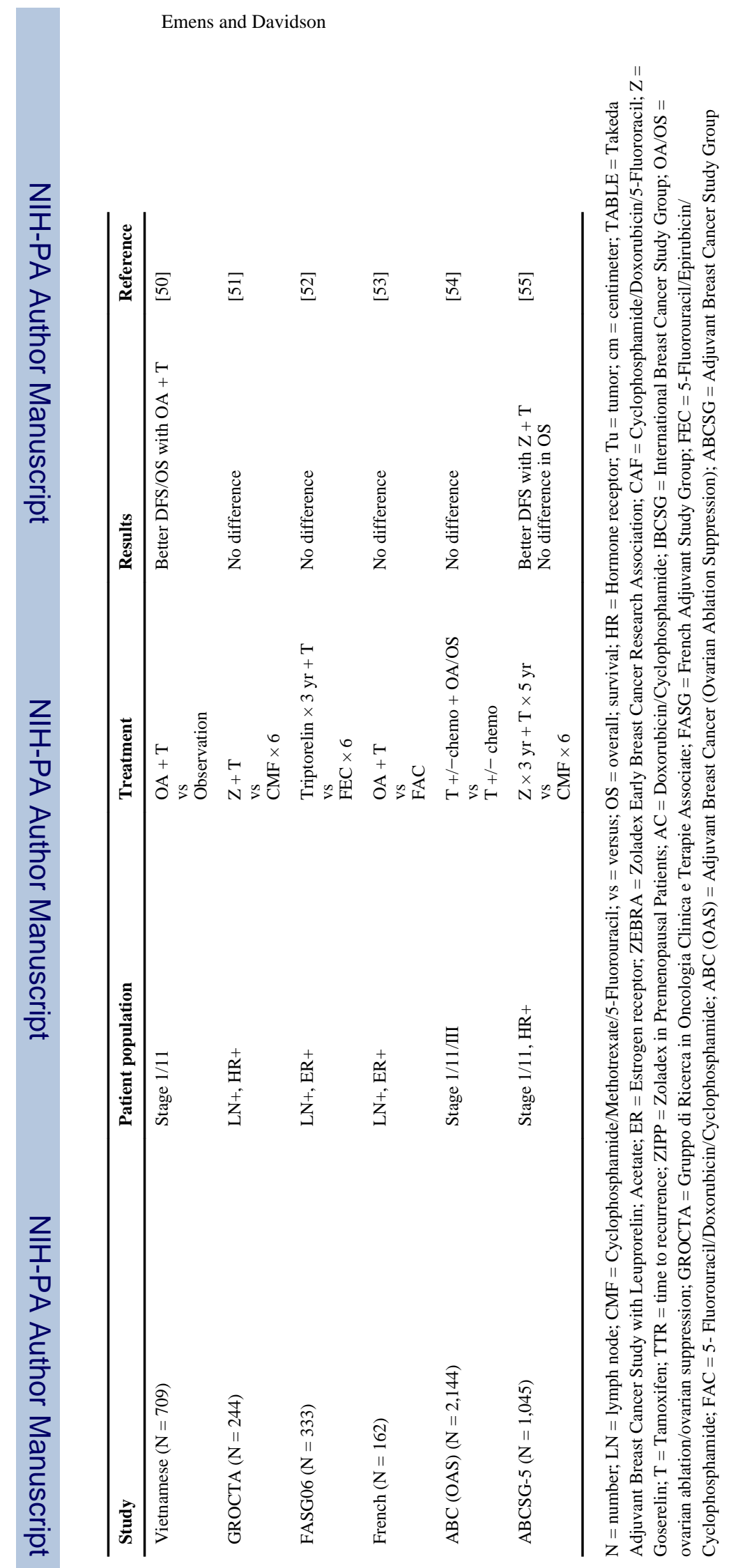

Cancer Treat Res. Author manuscript; available in PMC 2011 May 3. 
Table 3

\section{Ongoing clinical trials of ovarian ablation/suppression}

\begin{tabular}{|c|c|c|}
\hline Trial & Patient population & Intervention \\
\hline ABCSG-12 & $\begin{array}{l}\text { Premenopausal } \\
\text { Stage I/II/III } \\
\text { HR+ }\end{array}$ & $\begin{array}{l}G+A \\
v s \\
G+A+Z \\
v s \\
G+T \\
v s \\
G+T+Z\end{array}$ \\
\hline IBSCG 24-02/BIG 2-02: SOFT & $\begin{array}{l}\text { Premenopausal } \\
\text { HR+ } \\
\text { No adjuvant chemotherapy or premenopausal after adjuvant chemotherapy }\end{array}$ & $\begin{array}{l}\mathrm{T} \\
\text { vs } \\
\mathrm{OS}+\mathrm{T} \\
\mathrm{vs} \\
\mathrm{OS}+\mathrm{AI}\end{array}$ \\
\hline IBSCG 25-02/BIG 3-02: TEXT & $\begin{array}{l}\text { Premenopausal women with HR+ tumors who require OS with or without chemotherapy } \\
\text { from the start of adjuvant therapy }\end{array}$ & $\begin{array}{l}\mathrm{OS}+\mathrm{T} \\
\mathrm{vS} \\
\mathrm{OS}+\mathrm{AI}\end{array}$ \\
\hline
\end{tabular}

$\mathrm{ABCSG}=$ Adjuvant Breast Cancer Study Group; BIG = Breast International Group; HR = hormone receptor; $\mathrm{G}=$ Goserelin; $\mathrm{A}=$ anastrozole; $\mathrm{Z}=$ Zolendronate T $=$ Tamoxifen; $\mathrm{IBSCG}=$ International Breast Cancer Study Group; SOFT $=$ Suppression of Ovarian Function Trial; AI = aromatase inhibitor; TEXT $=$ Tamoxifen and Exemestane Trial 
Table 4

Trials comparing initial tamoxifen with aromatase inhibition

\begin{tabular}{|c|c|c|c|c|}
\hline Study & Patient population & Intervention & Results & Ref \\
\hline $\begin{array}{l}\text { ATAC } \\
(\mathrm{N}=9,366)\end{array}$ & Postmenopausal & $\begin{array}{l}\mathrm{A} \times 5 \mathrm{yr} \\
\mathrm{vs} \\
\mathrm{T} \times 5 \mathrm{yr} \\
\mathrm{vs} \\
\mathrm{A}+\mathrm{T} \times 5 \mathrm{yr}\end{array}$ & $\begin{array}{l}A>T \text { for } D F S \\
A+T=T\end{array}$ & {$[60,61]$} \\
\hline $\begin{array}{l}\text { BIG I-98 } \\
(\mathrm{N}=8,028)\end{array}$ & Postmenopausal ER+ and/or PR+ & $\begin{array}{l}\mathrm{T} \times 5 \mathrm{yr} \\
\mathrm{vs} \\
\mathrm{L} \times 5 \mathrm{yr} \\
\mathrm{vs} \\
\mathrm{T} \times 2 \mathrm{yr}, \mathrm{L} \times 3 \mathrm{yr} \\
\mathrm{vs} \\
\mathrm{L} \times 2 \mathrm{yr}, \mathrm{T} \times 3 \mathrm{yr}\end{array}$ & $\begin{array}{l}\mathrm{L}>\mathrm{T} \text { for } \mathrm{DFS} * \\
\mathrm{~L}>\mathrm{T} \text { for distant recurrence } \\
* \mathrm{~T} \text { compared to } \mathrm{L} \text { upfront only }\end{array}$ & {$[62,63]$} \\
\hline TEAM & Postmenopausal & $\begin{array}{l}\mathrm{T} \times 5 \mathrm{yr} \\
\mathrm{vs} \\
\mathrm{E} \times 5 \mathrm{yr} \\
\mathrm{vs} \\
\mathrm{T} \times 2 \mathrm{yr}, \mathrm{E} \times 3 \mathrm{yr} \\
\mathrm{vs} \\
\mathrm{E} \times 2 \mathrm{yr}, \mathrm{T} \times 3 \mathrm{yr}\end{array}$ & NR, ongoing & - \\
\hline
\end{tabular}

ATAC $=$ Arimidex, Tamoxifen, Alone or in Combination; $\mathrm{A}=$ Arimidex $; \mathrm{T}=$ Tamoxifen; DFS $=$ disease-free survival; $\mathrm{BIG}=\mathrm{Breast}$ International Group; $\mathrm{ER}=$ estrogen receptor; $\mathrm{PR}=$ progesterone receptor; $\mathrm{L}=$ Letrozole; $\mathrm{TEAM}=$ Tamoxifen and Exemestane Adjuvant Multicenter; $\mathrm{E}=$ Exemestane; $\mathrm{NR}=$ not reported 
Table 5

Trials testing aromatase inhibitors in sequence with tamoxifen

\begin{tabular}{lllll}
\hline Study & Patient population & Intervention & Result & Ref \\
\hline $\begin{array}{l}\text { Italian ITA } \\
(\mathrm{N}=426)\end{array}$ & Postmenopausal, HR+ & $\mathrm{T} \times 2 \mathrm{yr}, \mathrm{T} \times 3 \mathrm{yr}$ & $\mathrm{T}+\mathrm{A}>\mathrm{T}$ for RFS & {$[65]$} \\
& & $\mathrm{vs} \times 2 \mathrm{yr}, \mathrm{A} \times 3 \mathrm{yr}$ & & \\
$\begin{array}{l}\text { ABCSG-8/ARNO } \\
(\mathrm{N}=3,224)\end{array}$ & Postmenopausal, HR+ & $\mathrm{T} \times 2 \mathrm{yr}, \mathrm{T} \times 3 \mathrm{yr}$ & $\mathrm{T}+\mathrm{A}>\mathrm{T}$ for DFS, OS & {$[66,67]$} \\
& & $\mathrm{vs} \times 2 \mathrm{yr}, \mathrm{A} \times 3 \mathrm{yr}$ & & \\
$\begin{array}{l}\text { IES } \\
\text { (N=4,742) }\end{array}$ & Postmenopausal, ER+ and/or PR+ & $\mathrm{T} \times 2-3 \mathrm{yr}, \mathrm{T} \times 2-3 \mathrm{yr}$ & $\mathrm{T}+\mathrm{E}>\mathrm{T}$ for DFS, OS & [68, 69] \\
& & $\mathrm{vs} \times 2-3 \mathrm{yr}, \mathrm{E} \times 2-3 \mathrm{yr}$ & & \\
$\begin{array}{l}\text { NCIC-CTG MA.17 } \\
(\mathrm{N}=5,187)\end{array}$ & Postmenopausal ER+ and/or PR+ & $\mathrm{T} \times 5 \mathrm{yr}, \mathrm{P} \times 5 \mathrm{yr}$ & $\mathrm{T}+\mathrm{L}>\mathrm{T}$ for DFS & [70, 71] \\
& & $\mathrm{vs} \times 5 \mathrm{yr}, \mathrm{L} \times 5 \mathrm{yr}$ & $\mathrm{T}+\mathrm{L}>\mathrm{T}$ for OS if LN+ & \\
\hline
\end{tabular}

$\mathrm{HR}=$ hormone receptor $\mathrm{T}=$ Tamoxifen; $\mathrm{A}=$ Anastrozole RFS = relapse-free survival; $\mathrm{ABCSG}=$ Austrian Breast and Colorectal Cancer Study Group; ARNO = German Adjuvant Breast Cancer Group; DFS = disease-free survival; OS = overall survival; IES = Intergroup Exemestane Study Group; ER = estrogen receptor; PR = progesterone receptor; E = exemestane; NCIC-CTG MA. 17 = National Cancer Institute of Cancer Clinical Trials Group MA.17 Trial; P = placebo; L = Letrozole; LN = lymph node 